\title{
Corrosion Inhibition Study of Zinc Oxide-Polyaniline Nanocomposite for Aluminum and Steel
}

\author{
Farah Alvi $^{1, \text { * }}$, Naveed Aslam², Saleem Farooq Shaukat ${ }^{1}$ \\ ${ }^{1}$ Department of Physics, COMSATS Institute of Information Technology, Lahore 54000, Pakistan \\ ${ }^{2}$ GPS Innovations LLC, 12 Greenway Plaza Suites 1100, Houston, TX, USA 77046
}

Email address:

farahalvi@ciitlahore.edu.pk (F. Alvi)

\section{To cite this article:}

Farah Alvi, Naveed Aslam, Saleem Farooq Shaukat. Corrosion Inhibition Study of Zinc Oxide-Polyaniline Nanocomposite for Aluminum and Steel. American Journal of Applied Chemistry. Vol. 3, No. 2, 2015, pp. 57-64. doi: 10.11648/j.ajac.20150302.14

\begin{abstract}
The nanocomposites of inorganic oxide with conducting polymer display novel properties resulting from the molecular level interaction of the two dissimilar chemical components. Recently, Farah et al have synthesized ZnO-polyaniline (PANI) nanocomposite, and have studied the electronic properties of ZnO-PANI films [1]. Although to date, chromium has proved the best candidate in corrosion inhibition in acidic medium, but still it is hoped that the combination of different techniques and various compounds will provide the required protection against corrosion. Our present study has shown that the ZnO-PANI nanocomposite coatings on steel and aluminum prevents corrosion due to the internal sacrificial electrode formation and as well as the barrier effect at the ZnO-PANI and metal interface.
\end{abstract}

Keywords: Conducting Polymers, Corrosion, ZnO, Impedance, Tafel, Electrochemistry, Steel, Aluminum, Redox Potential

\section{Introduction}

The polyaniline exhibits environment stability, ease of synthesis and possesses several important properties that have been exploited as inhibitors in protective coatings materials [1-2]. Recently, copolymer based polyanilines have been found to yield a superior coating material for corrosion inhibition. The corrosion prevention has been enhanced using polyaniline combined with inorganic particles or clay platelets as a composite, i.e. PANI-montmorillonite (MMT), poly (o- methoxyaniline)-polyvinylchloride, Polyaniline-ZnO and nanodiamond-PANI etc. [3]. However, many reports have shown that polyaniline, topcoat polyaniline, or traditional topcoat combinational polyaniline adhere poorly [4]. Few reports have shown that emeraldine base or emeraldine base composite with polyvinylchloride inhibit corrosion better than emeraldine salt or doped form of polyaniline in the presence of $\mathrm{NaCl}$ solution. However, the results of the emeraldine salt form of polyaniline have been found to show better inhibition properties due to the electronic properties of conducting polymers, but similar non-conjugated polymers do not provide the same degree of protection. Zinc oxide has been widely used in cyclation as well as in selective oxidation of the heterogeneous and homogeneous systems [5]. Zinc oxide $(\mathrm{ZnO})$ is multifunctional semiconducting material due to the wide band gap $(3.37 \mathrm{eV}$ at $300 \mathrm{~K})$ and a large exciton binding energy $(60 \mathrm{meV}) . \mathrm{ZnO}$ is a suitable material to fabricate nanocomposite polymer scaffold due to its energy level, high electronic mobility, and environmental stability. Therefore $\mathrm{ZnO}$-conducting polymer nanocomposite films on different substrates have shown interesting anticorrosive properties.

The industrial need of aluminum and aluminum alloys are constantly expanding. Aluminum generally is light weight, cheap, good conductors of heat and electricity and corrosion resistive at moderate temperatures making it one of promising candidate for various applications. However the use of aluminum, despite of its inherent advantages, is still not utilized fully for many applications due to its significant corrosion behavior in aggressive environments containing chloride ions [6].

Steel has also been found the most exciting commodity metal from a technological point of view despite the growth in usage of aluminum. However, it must be protected against corrosion under normal environmental conditions. Stainless steel's is promising material for many applications due to resistance to corrosion and staining, low maintenance and relatively low cost. Due to the unique properties of stainless steel, find uses in exotic and highly-demanding applications, such as in the turbine blades of jet engines, in spacecraft, and in nuclear reactors. In order to meet with current and future 
environmental limitations, new effective and nontoxic coating systems are being searched. Protective coatings generally act as a barrier and isolate the corroding metal from the environment. The issue with these protective coatings is the acceleration of corrosion rate, if the coatings are having pinholes or any mechanical scratch due to small anode-large cathode configuration. Therefore the conducting polymers due to its own redox activity present in the coatings passivate the metal surface at pinhole areas immediately [7].

Hence present study has been carried out on the electrochemical investigation of $\mathrm{ZnO}-\mathrm{PANI}$ nanohybrid composite films coated on aluminum (99.9\%) and stain less steel type (304). The aim of this work is to determine the corrosion inhibition applicability of ZnO-PANI films on steel and aluminum immersed in salt $(\mathrm{NaCl})$ and acid $(\mathrm{HCl})$ environments. It is believed that nano-hybrid composites containing nano-additives would remarkably improve the substrate barrier properties.

\section{Experimental}

\subsection{Reagents and Materials}

Aniline (99.5\%), ammonium persulfate (98\%), sodium hydroxide (powder, 97\%) and hydrochloric acid (37\%) were all ACS grade and purchased from Sigma-Aldrich (USA). NN-dimethylformamide (99.8\%) was purchased from Alfa Aesar (USA). ZnO nanoparticles (99.8\%, dia 10-30 nm) were purchased from SRY Spring Nanomaterials Inc. All these chemicals and materials were employed as purchased without any further purification unless specified. The Stainless steel foil of type 304, having (composition of $\mathrm{Cr}=19 \mathrm{wt} \%$, $\mathrm{Ni}=11 \mathrm{wt} \%$ and $\mathrm{Fe}=70 \mathrm{wt} \%$ with thickness of $0.07 \mathrm{~cm}$ ) and aluminum $(99.9 \%, 0.025 \mathrm{~mm})$ were used in corrosion studies. Samples used in corrosion study were mechanically press cut into $2.5 \mathrm{~cm} \times 3.0 \mathrm{~cm}$ rectangular strip and used without any further polishing. However, they were, degreased in absolute ethanol, methanol, and propane and dried in Nitrogen to keep in moisture free desiccators before the corrosion studies were commenced [1-8].

\subsection{Synthesis and Coating of ZnO-PANI Nanocomposites Material}

The ZnO-PANI nanocomposite was chemically synthesized by the oxidative polymerization of aniline using ammonium peroxydisulfate $\left.\left[\left(\mathrm{NH}_{4}\right)_{2} \mathrm{~S}_{2} \mathrm{O}_{8}\right)\right]$ under controlled conditions [9]. The aniline to $\mathrm{ZnO}$ ratio was varied in the ratios $1: 1,3: 1,1: 3$ and added in $200 \mathrm{ml}$ of $1.0 \mathrm{M} \mathrm{HCl}$. This mixture was then cooled down to $4^{\circ} \mathrm{C}$ in an ice bath. Next, ammonium peroxydisulfate $(0.025 \mathrm{M})$ was dissolved in $100 \mathrm{ml}$ of $1.0 \mathrm{M} \mathrm{HCl}$ and Later, $\left.\left[\left(\mathrm{NH}_{4}\right)_{2} \mathrm{~S}_{2} \mathrm{O}_{8}\right)\right]$ in $1.0 \mathrm{M} \mathrm{HCl}$ solution was added dropwise to the above mentioned aniline solution and the reaction was continued stirring for 12 hours. The polymerization process was observed within ten minutes after the addition of ammonium peroxydisulfate as solution began to change color from light brown to green.

The dark green precipitate of the ZnO-PANI nanocomposite recovered from the reaction vessel was then filtered and washed using $1.0 \mathrm{M} \mathrm{HCl}$ (to remove any oxidant and oligomers). This precipitate was further washed using DI water, methanol and diethyl ether for the elimination of the low molecular weight polymer as well as oligomers. Finally the recovered precipitate was heated at $100^{\circ} \mathrm{C}$ for 6 hours in temperature-controlled oven. The dark green powder thus obtained was the emeraldine base form (EB) of the $\mathrm{ZnO}$ PANI nanocomposite. Further the emeraldine base form (EB) of the ZnO-PANI changed into emeraldine salt form (ES) by undoped in $1 \mathrm{M} \mathrm{NaOH}$ solution for 10 hours. In next step the dark blue precipitate of ZnO-PANI was obtained after filtering and several washing with methanol, acetone and DI water. Later, the ES form of ZnO-PANI powder was dissolved in N-methyl 2-pyrrolidinione (NMP) and was employed for the spin coating and film casting over stainless steel and aluminum substrates[1,10]. Average thickness of $\mathrm{ZnO}$-PANI coated on metal surfaces has kept at around 30-40 $\mu \mathrm{m}$. The thickness of the $\mathrm{ZnO}$-PANI film was measured using dektak profliometer.

\section{Results}

\subsection{Cyclic Voltammogram (CV)}

The corrosion set up consisted of an electrochemical cell comprised of three electrodes, $\mathrm{ZnO}$-PANI coated over the metal surface as the working electrode, platinum as the counter electrode, and $\mathrm{Ag} / \mathrm{AgCl}$ as the reference electrode. The over potential, CV, tafel and impedance of ZnO-PANI films were studied in $0.1 \mathrm{M} \mathrm{HCl}$ and $1 \mathrm{M} \mathrm{NaCl}$. All studies were done at room temperature. Figure $1(a, b)$ shows the cyclic voltammogram (CV) curves of $\mathrm{ZnO}$-PANI film coated over steel in $0.1 \mathrm{M} \mathrm{HCl}$ and $1 \mathrm{M} \mathrm{NaCl}$. The figure 1(a) displays the oxidation potential peaks at $-0.05 \mathrm{~V}$ and $0.67 \mathrm{~V}$ and reduction potential peaks at 0.76 and $0.05 \mathrm{~V}$ in $0.1 \mathrm{M} \mathrm{HCl}$. Nevertheless CV of ZnO-PANI film coated on steel in $1 \mathrm{M}$ $\mathrm{NaCl}$ solution in figure 1(b) depicts the oxidized potential at around $0.8 \mathrm{~V}$ indicating quasi-reversible system of $\mathrm{ZnO}-$ PANI in basic solution. The ZnO-PANI is oxidatively doped in contact with steel at $0.8 \mathrm{~V}$ in $1 \mathrm{M} \mathrm{NaCl}$ this result indicates that $\mathrm{ZnO}$-PANI in contact with steel can be anodically polarized. Interestingly P-type doped polymers provide a galvanic couple and the electrochemical potential of p-type doped polymers is determined by the chemical potential of the doped polymer and metal. Doped polymers with high chemical potentials when in contact with metals can strongly anodically polarized [11]. By comparison the $\mathrm{CV}$ response of $\mathrm{ZnO}$-PANI coated on steel in $0.1 \mathrm{M} \mathrm{HCl}$ shows larger surface area and larger potential window than in $1 \mathrm{M} \mathrm{NaCl}$ which simply ascribes the better reversibility and less ohmic resistance of system in acidic solution than in basic solution .

Following the above mentioned coating procedure a thick film was coated on aluminum $(99.9 \%)$ to ensure a uniform coating of the ZnO-PANI film. Comparatively to steel, current-voltage study for aluminum substrates have its own unique oxidation and reduction behavior for corrosion 
protection in $0.1 \mathrm{M} \mathrm{HCl}$ and $1 \mathrm{M} \mathrm{NaCl}$ as shown in figure 1 (c, d) in potential window of -0.4 to $1 \mathrm{~V}$. The $\mathrm{ZnO}-\mathrm{PANI} \mathrm{CV}$ response in figure (c) shows resistive effect of aluminum due to the formation of native oxide barrier on aluminum substrates in $0.1 \mathrm{M} \mathrm{HCl}$. whereas an oxidation peak has observed at $0.67 \mathrm{~V}$ for aluminum substrate in $1 \mathrm{M} \mathrm{NaCl}$. Due to the formation of native oxide barrier on aluminum substrate, the cyclic voltammogram in acidic solution shows more ohmic behavior than in steel. This in turn, can influence
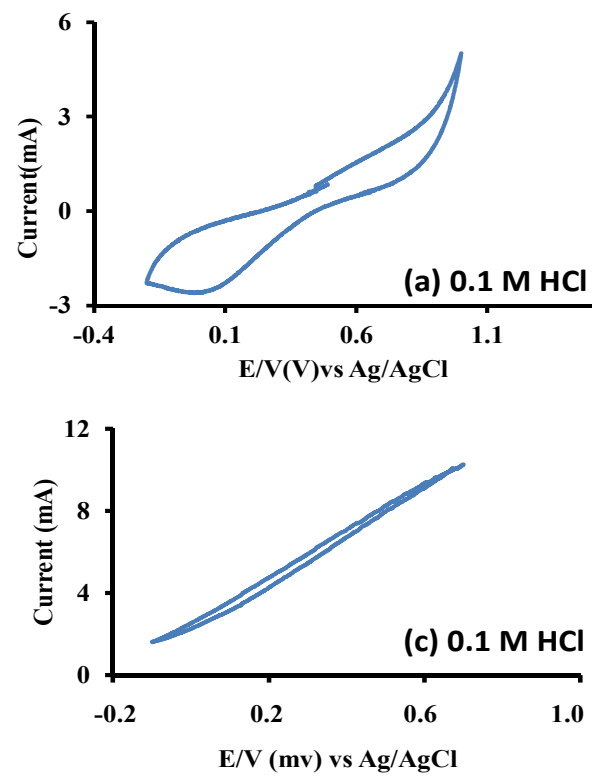

the degree to which a passivating metal may form a passivating oxide layer and heal (reform) this layer when scratched or damaged [12]. Based on the above discussion it has estimated that the existence of redox peaks simply illustrates that the $\mathrm{ZnO}$-PANI nanocomposite is active in acidic as well as in basic media. However the better anticorrosion capability of ZnO-PANI on steel substrate than in aluminum both in acidic and basic electrolytes has observed.
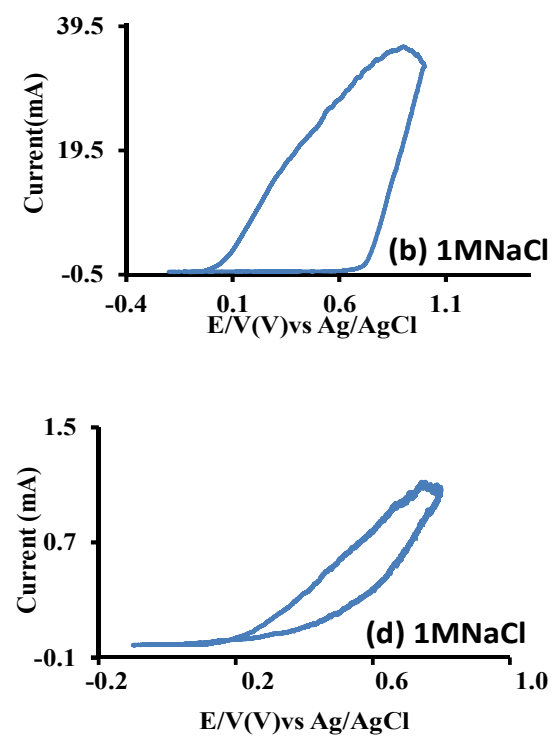

Figure 1. The CV of ZnO-PANI electrode coated over metal as working, Pt as counter electrode and Ag/AgCl as reference in an electrochemical cell where (a) film on steel in $0.1 \mathrm{M} \mathrm{HCl}$ (b) film on steel in $1 \mathrm{M} \mathrm{NaCl}$ (c) Film on over aluminum in $0.1 \mathrm{M} \mathrm{HCl}$ and (d) film on aluminum in $1 \mathrm{M} \mathrm{NaCl}$.

\subsection{Tafel Study}

Figure 2 (a,b,c,d) shows the tafel graph of $\mathrm{ZnO}-\mathrm{PANI}$ coated on steel and aluminum specimens in $0.1 \mathrm{M} \mathrm{HCl}$ and $1 \mathrm{M} \mathrm{NaCl}$ solution. The effect of the nature of the electrolyte could be easily distinguished from ZnO-PANI deposited on either steel or aluminum. However, shape of the tafel curves are similar, indicating that the ZnO-PANI film on steel and aluminum may act as a physical barrier, and enhance the corrosion resistance while reducing the migration rates of water and electrolytes at the interface of electrode and electrolyte without making any significant difference in the dynamics of electrode.

By comparison a typical ohmic behavior of ZnO-PANI has observed coated on aluminum than on steel in $0.1 \mathrm{M} \mathrm{HCl}$. It clearly indicates the high electronic and ionic conductivity of $\mathrm{ZnO}$-PANI film coated on steel than on aluminum substrate. However tafel study reveals a barrier effect of $\mathrm{ZnO}$-PANI in the $1 \mathrm{M} \mathrm{NaCl}$ electrolyte solution regardless of the substrate (steel or aluminum). However the linear polarization curves in figure 2 demonstrates the corrosion potential at $0.71 \mathrm{~V}$ of $\mathrm{ZnO}$-PANI coated on steel in $0.1 \mathrm{M} \mathrm{HCl}$. By comparison small corrosion current is noticed for steel sample than aluminum substrate either in $0.1 \mathrm{M} \mathrm{HCl}$ and $1 \mathrm{M} \mathrm{NaCl}$ at the corrosion potential of $0.71 \mathrm{~V}$. This attributes the better corrosion protection of $\mathrm{ZnO}-\mathrm{PANI}$ film on steel substrate than on aluminum regardless of electrolyte. Otherwise the better adhesion of $\mathrm{ZnO}$-PANI film on steel than on aluminum can also be the reason of providing improved corrosion protection with less corrosion current. Whereas high current density of ZnO-PANI coated on aluminum shows the interfacial barrier of aluminum oxide and poor adhesion property. Therefore above tafel discussion of ZnO-PANI illustrates the restricted mobility of electrolyte ions to the metal surface both in acidic and basic electrolytes which is in good agreement with the CV results already discussed in 3.1 . This is indicative that $\mathrm{ZnO}-\mathrm{PANI}$ nanocomposite matrix reflects the remarkable anticorrosion properties for steel and aluminum. Figure3 shows corrosion resistance ( $\mathrm{Rp}$ ) value of $\mathrm{ZnO}-\mathrm{PANI}$ for steel and aluminum specimens as a function of time. The polarization resistance which has been calculated using the tafel curves as described in equation1 [13].

$$
\mathrm{Rp}=\mathrm{I} / \mathrm{E}
$$

Where $\Delta \mathrm{E}$ is polarization potential and $\Delta \mathrm{I}$ is the polarization current.

Interestingly, the resistance has been found to increase due to passivation of $\mathrm{ZnO}-\mathrm{PANI}$ with steel and aluminum either in $\mathrm{HCl}$ or $\mathrm{NaCl}$ electrolytes. However the study of polarization resistance $(\mathrm{Rp})$ reveals the slightly decreasing resistance characteristics of polymer film as a function of time and found to be stable nearly for 2000 minutes. By 
comparison the $\mathrm{Rp}$ value of $\mathrm{ZnO}$-PANI has calculated for steel and aluminum as shown in figure 3 in $0.1 \mathrm{M} \mathrm{HCl}$ found nearly around $7000 \Omega$ and $5500 \Omega$. The higher polarization resistance of $\mathrm{ZnO}$-PANI for steel than aluminum is an evident of better protection of film with steel substrate both in acidic and basic solution. Besides the higher value of polarization resistance, rate of decrease in polarization resistance as a function of time is less for steel substrate than aluminum. For steel the Rp has become stable around 1200 minutes whereas for aluminum $\mathrm{Rp}$ got stable around 2200 minutes, this again shows the superior protective property of $\mathrm{ZnO}-\mathrm{PANI}$ coated on steel over aluminum. It might also be due to full coverage and improved interfacial contacts of ZnO-PANI film with steel than aluminum substrate. In addition to acidic the $\mathrm{Rp}$ value calculated for basic solution
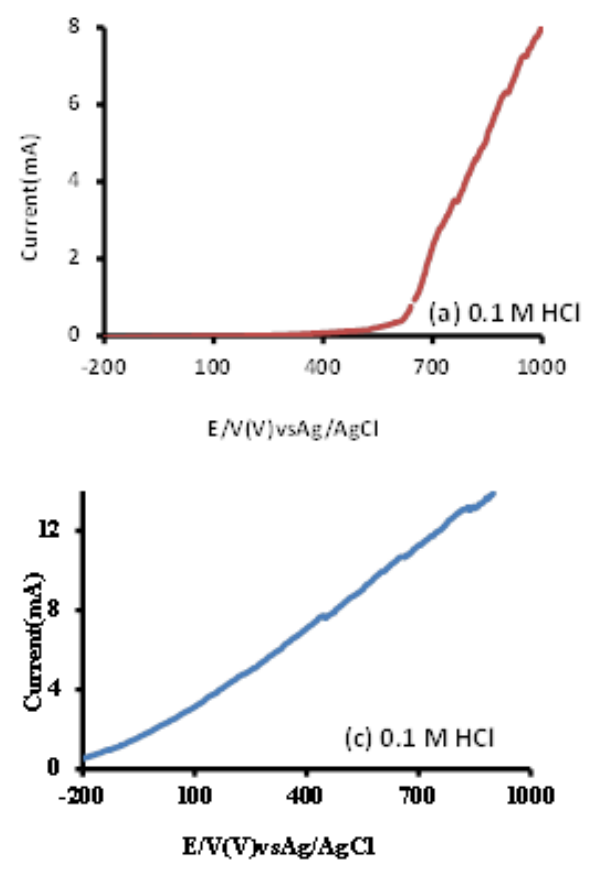

was also found higher for stainless-steel than aluminum (not shown here). Anticorrosion properties of ZnO-PANI coated on steel and aluminum has also been confirmed by measuring the open circuit potential in acidic and basic electrolytes. The inset in figure 3 gives comparison of the initial open circuit potential(OCP) of ZnO-PANI for stainless steel and aluminum which was observed at around $0.3 \mathrm{~V}$ and $0.2 \mathrm{~V}$ in $0.1 \mathrm{M} \mathrm{HCl}$ in the passive range, and was nearly stable over the course of 30 days of testing. Higher open circuit potential value of $\mathrm{ZnO}-\mathrm{PANI}$ film coated on stainless steel shows the higher corrosion resistance. Slight decrease in the (OCP) value over the period of time for steel and aluminum samples solidify the conformal and uniform coverage of ZnO-PANI on both substrates and their superior corrosion protection characteristics.
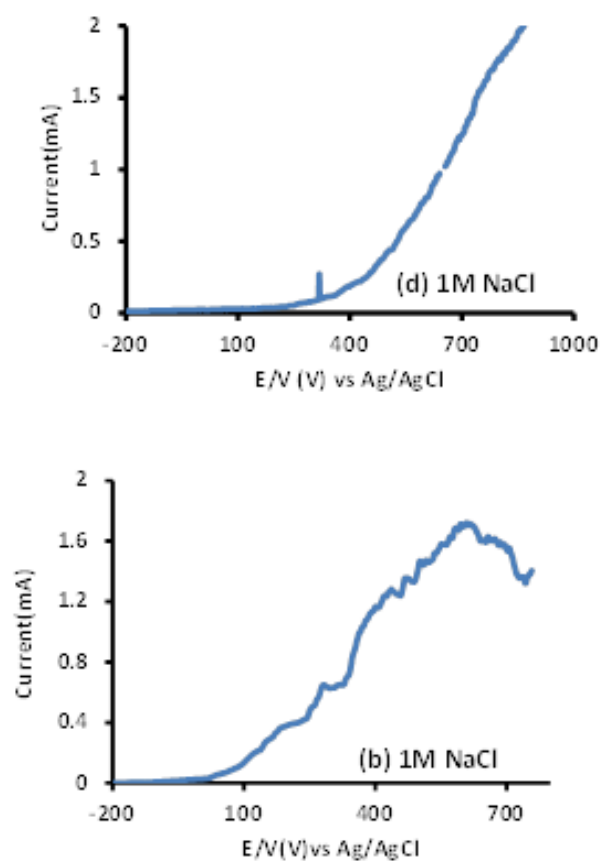

Figure 2. Tafel graph of ZnO-PANI coated over metal and in electrolytes: (a) film on steel in $0.1 \mathrm{M} \mathrm{HCl}$, (b) films on steel in $1 \mathrm{M} \mathrm{NaCl}$, (c) film on aluminum in $0.1 \mathrm{M} \mathrm{HCl}$ and (d) film on aluminum in $1 \mathrm{M} \mathrm{NaCl}$.

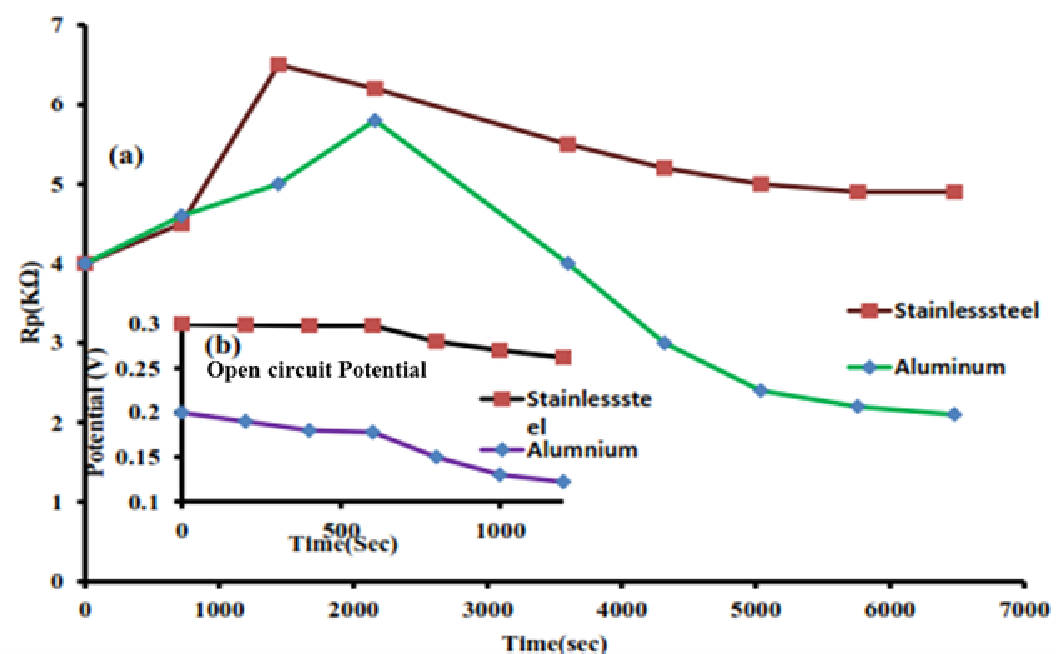

Figure 3. Rp variation of ZnO-PANI film coated over stainless steel and aluminum with time inO.1MHCl.(b)Open circuit potential variation with of ZnO-PANI film coated over stainless steel and Aluminum 


\subsection{Electrochemical Impedance Spectroscopy (EIS)}

The EIS method is used to monitor and predict the degradation of the coatings. The durability and performance of protective coatings in typical service environments depend to a large extent on the prevention of the substrate corrosion. A slow potentiometric scan method is used to measure the corrosion inhibitor efficiency of ZnO-PANI coated steel and aluminum samples. It measures the current as the potential (E) at the metal/solution interface that is scanned from a negative potential to the free corrosion potential to more positive value than the later at a relatively slow scan rate $(0.1 \mathrm{mV} / \mathrm{s})$. Comparison of polarization resistances for a bare and coated metal allows the calculation of the corrosion inhibitor efficiency (IE), which is used, to rate the different surface treatments [14]. The electrochemical impedance analysis was performed in $0.1 \mathrm{M} \mathrm{HCl}$ and $1 \mathrm{M} \mathrm{NaCl}$ electrolytes at room temperature in frequency range of $100 \mathrm{mHz}$ to $100 \mathrm{kHz}$. Figure $4(a, b)$ shows the nyquist plots of $\mathrm{ZnO}$-PANI coated on steel in $0.1 \mathrm{M} \mathrm{HCl}$ and $\mathrm{IM} \mathrm{NaCl}$ as a function of applied potential $(0 \mathrm{~V},-200 \mathrm{mV} .200 \mathrm{mV}, 400 \mathrm{mV})$ from high to low frequency. The impedance curve is divided in to three frequency region high, medium and low. The response of impedance curves of $\mathrm{ZnO}-\mathrm{PANI}$ in all three frequency regions are described as capacitive loop, capacitive loop and inductive loop. The impedance curves of $\mathrm{ZnO}-\mathrm{PANI}$ shows the high trend of semicircle formation coated on stainless steel than on aluminum in both electrolytes. Schematic of nature of impedance plots regarding to corrosion inhibition property is given in inset of figure 4. Plots shown in figure 4 reveal that when no potential is applied a typical semi-circle nature of the nyquist plot is observed. The presence of $\mathrm{ZnO}$ PANI in contact with the metal surface as well with the electrolyte $(0.1 \mathrm{M} \mathrm{HCl})$ undergoes a rapid oxidation process to provide a layer of $\mathrm{Fe}_{2} \mathrm{O}_{3}$ at the polyaniline/iron interface. Figure 5 shows the nyquist plot of $\mathrm{ZnO}$-PANI coated over aluminum in (a) $0.1 \mathrm{M} \mathrm{HCl}$ and (b) $1 \mathrm{M} \mathrm{NaCl}$ in a three electrode systems where $\mathrm{Pt}$ as counter electrode and $\mathrm{Ag} / \mathrm{AgCl}$ as the reference electrode. The ZnO-PANI coated over aluminum does not show the significant semicircular behavior compare to stainless steel in $0.1 \mathrm{M} \mathrm{HCl}$. This is consistent with the tafel and $\mathrm{CV}$ results for aluminum which mostly explains the ohmic behavior of ZnO-PANI film for aluminum. Figure 4 (b) shows the semicircle with interfacial polarization in presence of $1 \mathrm{M} \mathrm{NaCl}$. On comparing figure 4 and 5 it is clearly seen that $\mathrm{ZnO}-\mathrm{PANI}$ provides better corrosion inhibition in steel than aluminum in presence of $0.1 \mathrm{M} \mathrm{HCl}$ than in $1 \mathrm{M} \mathrm{NaCl}$. It attributes the superior anticorrosion property of $\mathrm{ZnO}-\mathrm{PANI}$ coated on stainless steel as well as on aluminum samples [15-16].

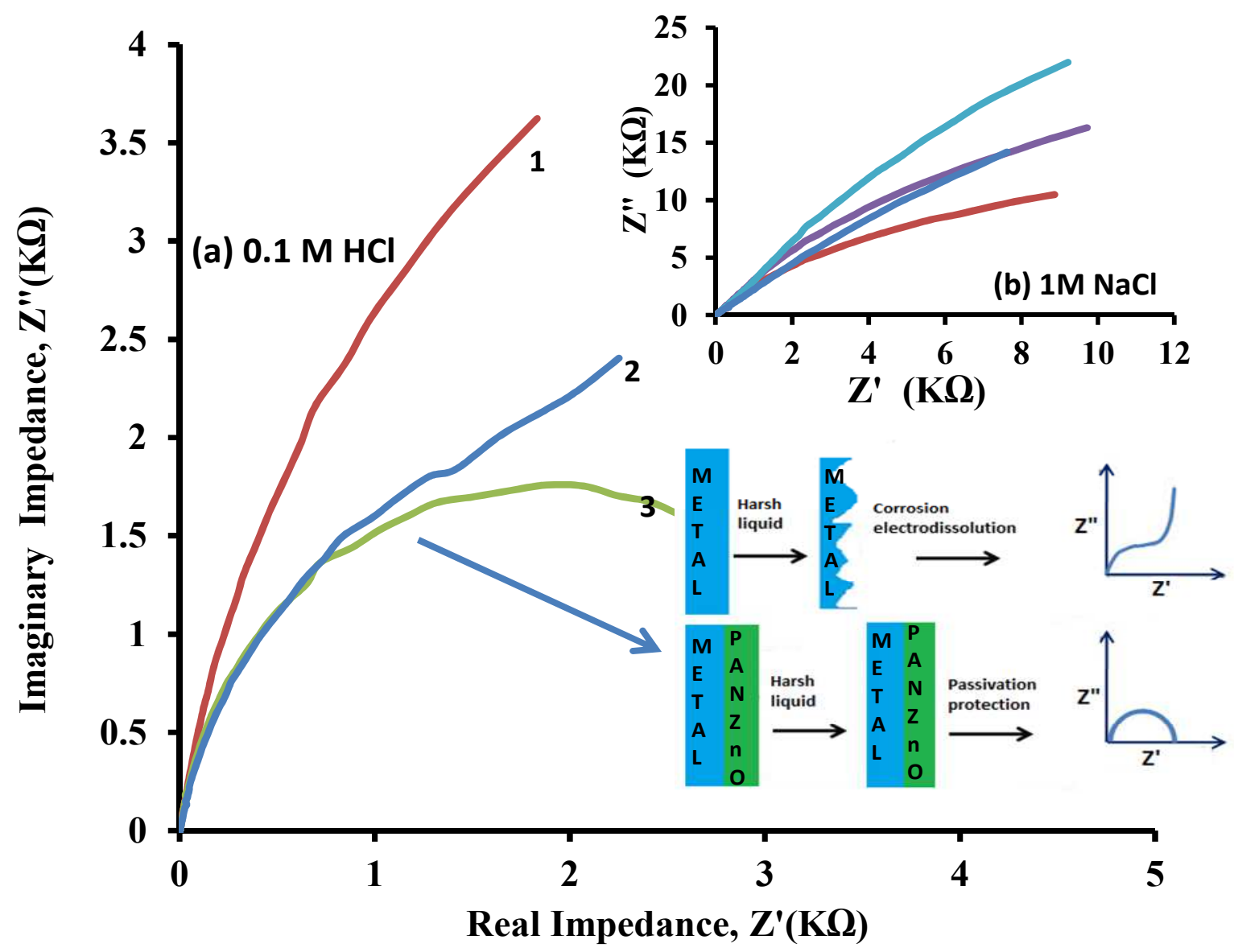

Figure 4. Nyquist plot of ZnO-PANI coated over steel in $0.1 \mathrm{M} \mathrm{HCl}(1,2,3)$ and right showing the schematic of corrosion inhibition due to Nyquist study and inset shows the Nyquist of ZnO-PANI deposited on steel in $1 \mathrm{M} \mathrm{NaCl}(a, b, c, d)$ as a function of applied potential (0V, -200 mV. $200 \mathrm{mV}, 400 \mathrm{mV})$. 

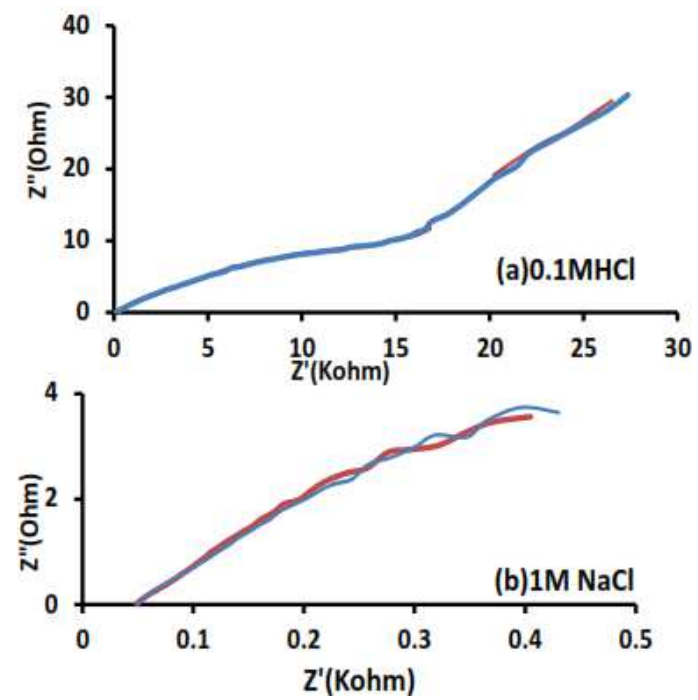

Figure 5. Nyquist plot of ZnO-PANI film coated over aluminum in (a) $0.1 \mathrm{M} \mathrm{HCl}$ and (b) $1 \mathrm{M} \mathrm{NaCl}$ in three electrode systems where Pt as counter and Ag/AgCl as reference electrode.

\subsection{SEM Studies}

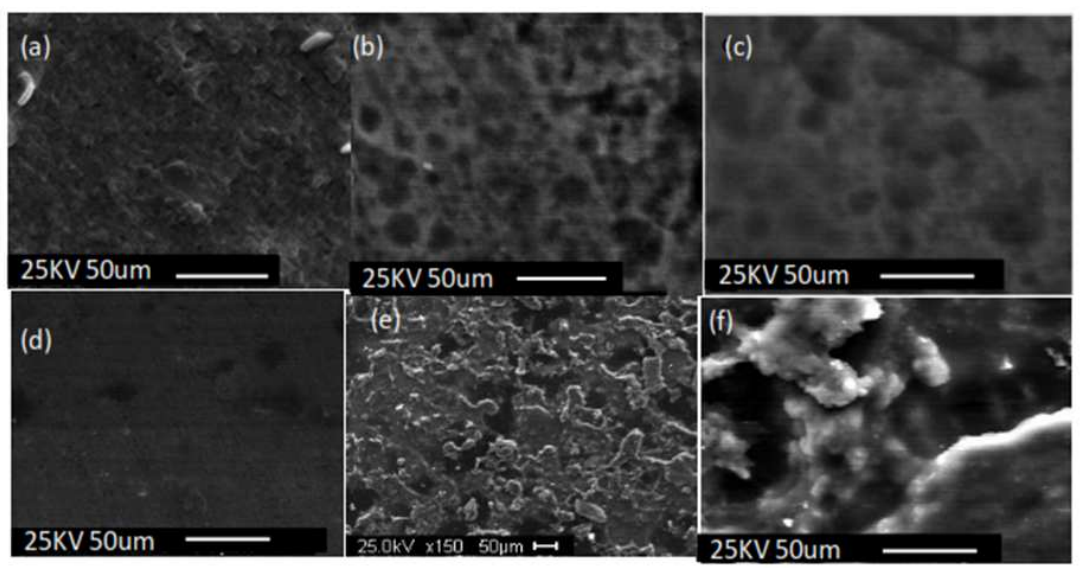

Figure 6. SEM pictures of ZnO-PANI nanocomposite on steel: (a) as made, and (b) tested in $0.1 \mathrm{HCl}$ solution (c) in $1 \mathrm{MNaCl}$ on stainless steel. (d, e, f) as made, in $0.1 \mathrm{MHCl}$ and $1 \mathrm{MNaCl}$ on aluminum.
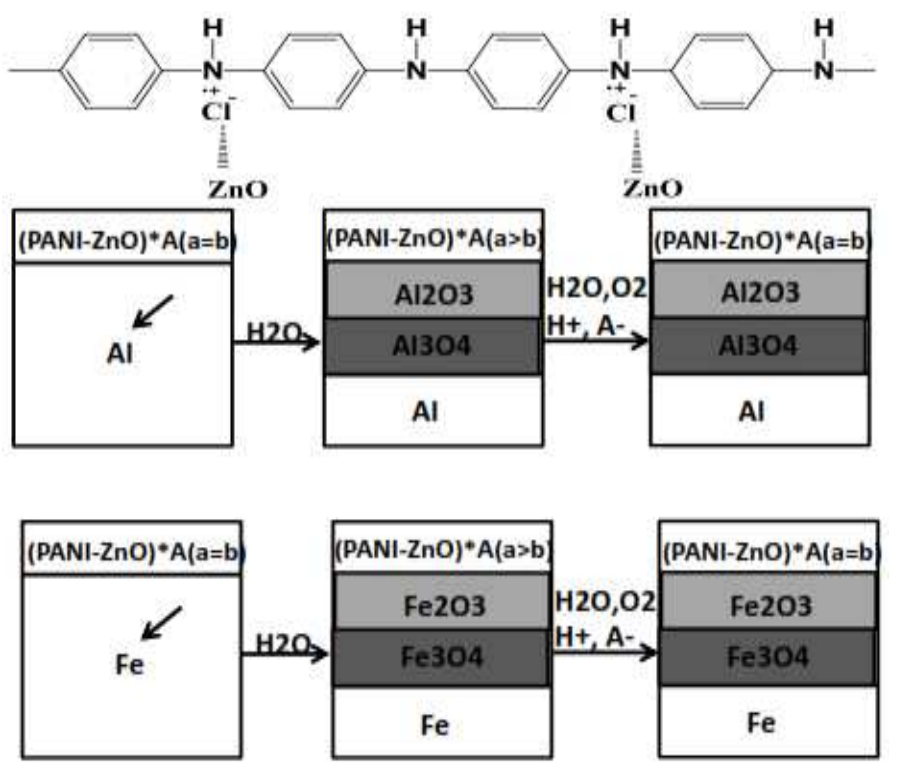

Figure 7. Schematic of ZnO-PANI nanocomposite on Aluminum and steel substrates for corrosion protection. 
Figure 6 (a.b.c.d.e.f) shows SEM pictures of $\mathrm{ZnO}-\mathrm{PANI}$ coated over steel and aluminum substrates for corrosion study in $0.1 \mathrm{M} \mathrm{HCl}$ and $1 \mathrm{M} \mathrm{NaCl}$ solution. Figure $6(\mathrm{a}, \mathrm{b}, \mathrm{c})$ shows SEM images of ZnO-PANI coated over stainless steel (a) before immersing in electrolyte (b) in $0.1 \mathrm{M} \mathrm{HCl}$ and (c) $1 \mathrm{M} \mathrm{NaCl}$. The surface morphology of the ZnO-PANI films changes slightly either exposed in $0.1 \mathrm{M} \mathrm{HCl}$ or $1 \mathrm{M} \mathrm{NaCl}$ solutions. However, no pin holes were observed in the film during the corrosion study for period of one month. There is certainly, the change in the structure of ZnO-PANI due to the flux in and out movement of the ions in the film as observed in figure $6(a, b)$. Similarly the figure $6(d, e, f)$ depicts the nearly same morphology of $\mathrm{ZnO}-\mathrm{PANI}$ film when coated on aluminum substrate. Figure $6(\mathrm{~d}, \mathrm{e}, \mathrm{f})$ shows the change in $\mathrm{ZnO}-\mathrm{PANI}$ morphology as (c) before immersing in electrolyte (d) in $0.1 \mathrm{M} \mathrm{HCl}$ and (e) $1 \mathrm{M} \mathrm{NaCl}$. Again no significant difference has observed in film morphology while using acidic and basic solution to examine the corrosion study for aluminum. However little scratching and peeling of film in the ZnO-PANI film coated on aluminum was observed over the period of time while immersing electrode in electrolytes. This information clearly supports that stainless steel substrate has better film adhesion and coverage due to the absence of native oxide layer as found in aluminum.

Figure 7 shows also a schematic of the ZnO-PANI in corrosion inhibition for stainless steel and aluminum specimens. It has been known that $\mathrm{Fe}_{3} \mathrm{O}_{4}$ composition is the passivation layer formed by PANI, so it is observed that PANI coatings on active metals like iron provide anodic protection, and act as electronic, chemical, and physical barriers to inhibit anodic reaction, and maintain high resistance to ionic flow, thereby blocking the metal dissolution and providing excellent corrosion protection. Besides, ZnO-PANI has also shown promising inhibition properties for aluminum due to the formation of underneath aluminum oxide layer $\left(\mathrm{Al}_{2} \mathrm{O}_{3}\right)$ which increases the stability of the material in higher potential. The ability of an oxide layer to prevent corrosion depends on its electrical resistivity. The higher the resistance of the oxide layer is the more effective it will be in preventing electron transfer. So from above discussion it can be understood that $\mathrm{ZnO}$-PANI reaches a steady state with oxygen, and passivated metal oxide layer is supposed to be regenerating rather than being consumed on both steel and aluminum.

\section{Conclusion}

The detailed study on corrosion inhibition properties of $\mathrm{ZnO}$-PANI nanocomposite was investigated using tafel, electrochemical impedance and cyclic voltammetry studies, respectively. The $\mathrm{CV}$ studies indicated that $\mathrm{ZnO}$-PANI in contact with steel could serve better than aluminum in $0.1 \mathrm{M}$ $\mathrm{HCl}$. The presence of $\mathrm{ZnO}-\mathrm{PANI}$ in contact with the metal surface as well with the electrolyte $(0.1 \mathrm{M} \mathrm{HCl})$ undergoes a rapid oxidation process providing a layer of $\mathrm{Fe}_{2} \mathrm{O}_{3}$ at polyaniline/iron interface. The studies indicated that electrochemical behavior of the PANI coatings had some important differences between stainless steel 304 and the aluminum (99\%). Based on the obtained corrosion currents, it is possible to believe that the thickness of the surface protective aluminum oxide and iron oxide layer plays a key role in the inhibiting effect.

The ZnO-PANI takes advantage of the excellent corrosion inhibitor characteristics of steel and aluminum due to its chain conformation and electronic properties. The work is in progress at our laboratory to use ZnO-PANI as corrosion inhibition layers for copper in corrosive environment.

\section{Acknowledgement}

Author highly appreciates the support of this work by NSF grant \# 0728100.

\section{References}

[1] F. Alvi, M.K .Ram, H. Gomez, R. K. Joshi and A. Kumar, "Evaluating the chemio-physio properties of novel zinc oxidepolyaniline nanocomposite polymer films" Polymer journal,2010, 42, 935-940.

[2] S. P. Sitaram, J. O. Stoffer, and T. J. O'Keefe, "Application of conducting polymers in corrosion protection," Journal of Coatings Technology, 1997, 69, 65-69.

[3] F. Mansfeld, "Use of electrochemical impedance spectroscopy for the study of corrosion protection by polymer coatings," Journal of Applied Electrochemistry, 1995, 25, 187-202.

[4] T. Schauer, A. Joos, L. Dulog, and C.D. Eisenbach, "Protection of iron against corrosion with polyaniline primers," Progress in Organic Coatings, 1998, 33, 20-27.

[5] U. Rammelt and G. Reinhard, “Applicant ion of electrochemical impedance spectroscopy (EIS) for characterizing the corrosion-protective performance of organic coatings on metals," Progress in Organic Coatings, 1992, 21, 205-226.

[6] T. Monetta, F. Bellucci, L. Nicodemo, and L. Nicolais, "Protective properties of epoxy based Organic coatings on mild steel, "Progress in OrganicCoatings, 1993, 21, 353-369.

[7] N. Yoichiro, M. Aki, K. Hiroko, A.Toru, H. Yoshinori and S. Goro, "Preparation of $\mathrm{ZnO}$ thin films for high-resolution field emission display by electron beam evaporation". Appl. Surf. Sci, 1999, 142, 233-236.

[8] Z. X. Xu, V. A. L Roy, P. Stallinga, M. Toffanin, H. F. Xiang, and C. M.Che, "Nanocomposite field effect transistors based on zinc oxide/polymer blends".Appl. Phys.Lett, 2007, 90, 223509- 2740478.

[9] G. Gustafsson, Y. Cao, G . M. Treacy, F. Klavetter, N. Colaneri, and A. Heeger, "Flexible light-emitting diodes made from soluble conducting polymers" Nature, 1992, 357, 477479.

[10] M. J. Sailor, E. J. Ginsburg, C. B. Gorman, A. Kumar, R. H. Grubbs, \& N. S. Lewis, Thin Films of n-Si/Poly-(CH3) SiCyclooctatetraene: Conducting-Polymer Solar Cells and Layered Structures. Science, 1990, 249, 1146-1149. 
[11] N. I. Kovtyukhova, A. D. Gorchinskiy, \& C. Waraksa, SelfAssembly of Nanostructured Composite $\mathrm{ZnO} /$ Polyaniline Films. Mater. Sci. Eng. B, 2000, 424, 69-70.

[12] J. E. O. Mayne, "The mechanism of the inhibition of the corrosion of iron and steel bemoans of paint," Official Digest, $1952,24,127-136$.

[13] B. N. Grgur, M. M. Gvozdenovi'c, V. B. Mi`skovi'cStankovi'c, and Z. Ka carevi'c-Popovi'c, "Corrosion behavior and thermal stability of electrodeposited PANI/epoxy coating system on mild steel in sodium chloride solution," Progress in Organic Coatings, 2006, 56, 214-219.
[14] V. Karpagam, S. Sathiyanarayanan, and G. Venkatachari, "Studies on corrosion protection of Al2024 T6 alloy by electropolymerized polyaniline coating," Current Applied Physics, 2008, 8, 93-98.

[15] P. R. P. Rodrigues, J. O. Zerbino, and S. M. L. Agostinho, "Voltammetric and ellipsometric studies of films formed on 304 stainless steel in sulphuric acid solution without and with benzotriazole," Materials Science Forum, 1998, 289, 12991310 .

[16] F. E.Taib Heakal and S. Haruyama, "Impedance studies of the inhibitive effect of benzotriazole on the corrosion of copper in sodium chloride medium," Corrosion Science, 1980, 20, 887898. 